\title{
Alterations in microRNA expression profiles in inflamed and non-inflamed ascending colon mucosae of patients with active Crohn's disease
}

Lu Yi Wu, ${ }^{1,2+}$ Xiao Peng Ma, ${ }^{2+}$ Yin Shi, ${ }^{2}$ Chun Hui Bao, ${ }^{2}$ Xiao Ming Jin,,${ }^{3}$ Yuan Lu, ${ }^{4}$ Ji Meng Zhao, ${ }^{2}$ Ci Li Zhou, ${ }^{2}$ Dai Chen, ${ }^{5}$ Hui Rong Liu ${ }^{2 *}$

${ }^{1}$ Qigong Institute, Shanghai University of Traditional Chinese Medicine, Shanghai, China; ${ }^{2 K e y}$

Laboratory of Acupuncture and Immunological Effects, Shanghai University of Traditional Chinese

Medicine, Shanghai, China; ${ }^{3}$ Stark Neurosciences Research Institute, Indiana University School of

Medicine, Indianapolis, IN, USA; ${ }^{4}$ Department of Mechanics and Engineering Science, Fudan

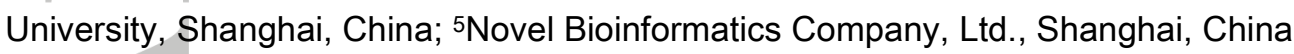

\section{Corresponding author:}

Hui Rong Liu, MD, PhD, E-mail: Ihr tcm@139.com / 13816024323@139.com

Tel.: +862164644238

Fax: +862164644238

Key Laboratory of Acupuncture and Immunological Effects, Shanghai University of Traditional Chinese Medicine, Shanghai 200030, China.

Running title: microRNAs Expression in Colon Mucosae of Patients

Conflicts of interest: None.

t'The first two authors (Lu Yi Wu, and Xiao Peng Ma) contributed equally to this work.

The abstract was previously presented at the Digestive Disease Week, San Diego, USA, May 21-24, 2016.

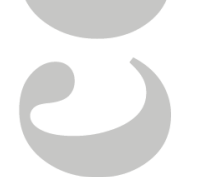

This article has been accepted for publication and undergone full peer review but has not been through the copyediting, typesetting, pagination and proofreading process which may lead to differences between this version and the Version of Record. Please cite this article as doi: $10.1111 /$ jgh.13778

This is the author's manuscript of the article published in final edited form as:

Wu, L. Y., Ma, X. P., Shi, Y., Bao, C. H., Jin, X. M., Lu, Y., Zhao, J. M., Zhou, C. L., Chen, D., and Liu, H. R. (2017) Alterations in microRNA expression profiles in inflamed and non-inflamed ascending colon mucosae of patients with active Crohn's disease. Journal of Gastroenterology and Hepatology, Accepted Author Manuscript. http://dx.doi.org/10.1111/jgh.13778. 
Abbreviations: $C D$, Crohn's disease; AM, Acupuncture and moxibustion; FC, fold chang; FDR, false discovery rate; GO, Gene Ontology; IBD, inflammatory bowel disease; iCD, inflammed Crohn's disease; niCD, non- inflammed Crohn's disease; qRT-PCR, quantitative real time polymerase chain reaction; miRNA, microRNA; UTR, untranslated region.

\section{Acknowledgements}

The authors are grateful to the patients who agreed to participate in the research. We also wish to thank Yun Hua Cui and Xiao Ming Feng for their assistance in the recruitment of subjects and data acquisition and Jie Zong of the Novel Bioinformatics Company (Shanghai, China) for his help with data analysis. This project was supported by funding from the Shanghai Rising-Star Program [No. 16QA1403400]; the National Key Basic Research Program of China [973 program: No. 2009 CB522900 and No. 2015CB554501]; the Shanghai Municipal Education Commission Research Fund for Outstanding Young Teachers [No. zzszy12011] New Century Excellent Talents in University [No. NCET-13-0907]; and the Shanghai Health System outstanding academic leader [No. XBR2013106]. Microarray experiments were performed by Kang Chen Bio-tech, Shanghai, China.

Author contributions: study concept and design: WLY, MXP and LHR; acquisition of data: WLY, JXM,

ZCL, LY, ZJM; statistical analyses: CD, LHR, BCH, WLY; drafting of the manuscript: WLY; revision of the manuscript: SY, LHR, MXP; study supervision: LHR. All authors read and approved the final manuscript.

This article is protected by copyright. All rights reserved. 


\section{Abstract}

Background and aims: The miRNA expression profiles of the terminal ileum, sigmoid colon, and rectal mucosa of adult patients with active Crohn's disease (CD) have been previously reported. The purpose of this study was to identify dysregulated miRNAs in the mucosa of the ascending colon.

Methods: Biopsy tissue samples were taken from the mucosae of inflammatory (iCD) or non-inflammatory (niCD) areas of the ascending colons of adult patients with active CD. miRNA and mRNA expression profiles were detected using microarray analyses. miRNAs and mRNAs demonstrating significant differences were validated via quantitative real-time PCR (qRT-PCR). Luciferase reporter genes were used to measure two miRNAs inhibition of potential target genes in human 293T cells in vitro.

Results: Compared with the HC group, the ascending colon miRNA expression profiles revealed that 43 miRNAs were significantly up-regulated and 35 were down-regulated in the iCD group. The mRNA expression profiles indicated that 3,370 transcripts were significantly differentially expressed in the ascending colon, with 2169 up-regulated and 1201 down-regulated mRNAs in the iCD group, and only 20 miRNAs demonstrated significant differential expression in the niCD group. In contrast, nearly 100 miRNAs significantly varied between the $\mathrm{iCD}$ and niCD groups. Finally, luciferase reporter gene assays showed that hsa-miR-16-1 directly regulated the human C10orf54 gene and that they were negatively correlated.

Conclusions: Our results indicated that the differentially expressed miRNAs and mRNAs were related to immune inflammation and intestinal flora. The data provide preliminary evidence that the occurrence of CD involves the inhibition of C10orf54 expression by hsa-miR-16-1.

Key Words: Crohn's disease; ascending colon mucosa; microRNA expression profiles; mRNAs 


\section{Introduction}

Inflammatory bowel disease (IBD) is an intestinal inflammatory disease characterized by chronic nature, and tendency to relapse. CD has a long and persistent disease duration, is recurrent, and can seriously affect the quality of life of patients. Therefore, CD has been designated by the World Health Organization (WHO) as an intractable digestive disease. In Europe, there are approximately 2.5-3 million IBD patients, costing approximately $€ 4.6-5.6$ billion in healthcare spending each year ${ }^{1}$. The general clinical symptoms of Chinese patients are relatively mild: there are fewer incidences of symptoms outside of the intestine as well as fewer complications, and fistula and perianal complications are rare ${ }^{2}$. However, with the recent improvement of China's GDP and quality of life, the incidence of CD clearly trended upward, the age of onset has tended to be younger ${ }^{2}$, and familial aggregation has become less significant ${ }^{3}$. Although it is currently considered that the pathogenesis and development of $\mathrm{CD}$ is closely related to interactions between the internal and external environments of the body, susceptibility genes, and immunomodulatory factors, the precise pathogenic mechanism remains unclear ${ }^{4,5}$.

In the past 20 years, international collaboration via genome-wide association studies (GWAS) investigated potential IBD pathogenic genes. In these studies, CD occurrence was closely related to autophagy and immunity ${ }^{6}$. miRNAs are small non-coding RNAs (21-23 nucleotides in length) that bind to specific target mRNAs, thereby post-transcriptionally inhibiting gene expression ${ }^{7,8}$. Although the biological functions of only a small portion of the miRNAs have been elucidated, their importance is exemplified by roles in the regulation of gene expression, the cell cycle, and development as well as in immune response regulation ${ }^{7,9}$. miRNA binding sites are often located in the $3^{\prime}$ untranslated region 
(UTR) of a target mRNA transcript, leading to mRNA degradation or translational inhibition. An increasing number of studies have focused on miRNA function in the intestinal immune system: these results provide evidence that miRNAs can regulate intestinal epithelial tight junction permeability ${ }^{10}$ and $\mathrm{T}$ helper cell (Th)-17-mediated inflammation and autophagy ${ }^{11,12}$. Second-generation sequencing methods have been applied to active CD patient samples versus healthy control groups to measure differential miRNA expression profiles in the peripheral blood and inflammatory and non-inflammatory areas of intestinal tissues ${ }^{13,14}$. However, few studies have focused on miRNA expression in the mucosae of affected areas (the terminal ileum, sigmoid colon, and rectum) in active CD patients ${ }^{15-17}$, the ascending colon and the terminal ileum are the most common sites of $\mathrm{CD}^{18}$, protein biomarkers of ascending colon biopsies may differentiate Crohn's disease from UC throughout proteomic analysis ${ }^{19}$, and no reports have investigated miRNA mechanisms from the perspective of the ascending colon mucosa of CD patients. Hence, the miRNA expression profile characteristics of the ascending colon mucosa remain unclear.

The goal of this study was to observe whether miRNA expression profiles were altered in the ascending colon mucosa of active $\mathrm{CD}$ patients and if these profiles differed between mucosa originating from inflammatory and non-inflammatory regions. We further sought to identify differentially expressed mRNAs in inflammatory ascending colon mucosae from CD patients. 


\section{Materials and Methods}

\section{Subjects and ascending colon tissue collection}

Adult patients were come from the CD specialty department at the Shanghai Institute of Acupuncture and Meridian and Zhongshan Hospital of Fudan University from July 2009 to May 2010.

All patients were type L2B1 (colonic, non-stricturing, non-penetrating) and were enrolled according to the Montreal classification criteria ${ }^{20}$. Patients were evaluated using the Crohn's Disease Activity Index (CDAI), and all enrolled patients had CDAI scores greater than 150 points. A portion of biopsy specimens $\uparrow$ were from ascending colon regions with inflammation $(\mathrm{CD}$, ulcer surface, three biopsies/area), and the others were from normal regions $(\mathrm{CD}, 8 \mathrm{~cm}$ above the inflammatory mucosa, three biopsies/area). Healthy volunteers who were screened and provided colon biopsies were designated the healthy control (HC) group. HC and CD biopsy specimens were collected into pre-labelled cryopreservation vials, immediately soaked in liquid nitrogen, and stored at $-80{ }^{\circ} \mathrm{C}$ until testing. All patients and healthy volunteers were from a randomized controlled clinical trial (Clinical Trial Registration: ChiCTR-TRC-10000950), which was approved by the Ethics Committee of the Yueyang Hospital of Integrated Traditional Chinese and Western Medicine in affiliation with the Shanghai University of Traditional Chinese Medicine (No: 2009-02); all subjects provided informed consent. The methods were carried out in accordance with the approved guidelines 


\section{RNA extraction and quality control}

TRIzol reagent (Invitrogen, Carlsbad, CA, USA) was used to extract total RNA from the biopsy samples. The miRNeasy mini kit (QIAGEN, Hilden, Germany) was also used for miRNA separation and purification. A NanoDrop (ND-1000; NanoDrop Technologies, Wilmington, DE, USA) was used for quality determination and quantification of total RNA, and RNA integrity was observed following 1\% agarose gel electrophoresis. The Agilent 2100 (Agilent Technologies, Palo Alto, CA, USA) was used for quality control. Total RNA samples with an RNA integrity number (RIN) greater than 7 were used for microarray analysis.

\section{miRNA microarray analysis}

Shanghai KangChen Bio-tech measured the miRNA expression profiles of the ascending colon mucosa samples using the miRCURY ${ }^{\mathrm{TM}}$ LNA Array (v.16.0, Exiqon, Vedbaek, Denmark). The miRNA hybridization method was based on the procedures provided with the miRCURY LNA. RNA samples were labelled with Hy3 fluorescence using the miRCURY ${ }^{\mathrm{TM}}$ array labelling kit (Exiqon, Vedbaek, Denmark). Fluorescence-labelled miRNAs were identified after hybridization by sequencing. The hybridized miRNAs were washed using the buffer kit (Exiqon, Vedbaek, Denmark),

dried, and scanned using a GenePix 4000B array scanner (Molecular Devices, Sunnyvale, CA, USA).

\section{Gene microarray analysis}

Total RNA from each group was reverse transcribed into cDNA then labelled with Cy3. The labelled cDNA samples were hybridized with Homo sapiens arrays (Roche NimbleGen, No. 05543789001) containing 45,034 human genes. A GenePix 4000B microarray scanner scanned the 
monochromatic NimbleGen arrays, and GenePix Pro V6.0 was used to read the original image pixel. Details of the labelling, hybridization, scanning, and standardization are as described on the NimbleGen website (http://www.nimblegen.com). The standardized values were analysed using the online sequence analysis system GeneSifter (http://www.genesifter.net).

\section{miRNA and mRNA real-time quantitative PCR validation}

The miRNA and mRNA microarray results were verified via quantitative real-time PCR (qRT-PCR) of miRNA and mRNA expression in each group of ascending colon mucosal tissues. cDNA was synthesized in the presence of a dNTP mixture (HyTest, Turku, Finland) and MMLV reverse transcriptase (Epicentre, Madison, WI, USA) using the Gene Amp PCR System 9700 (Applied Biosystems). qRT-PCR was then conducted using the ABI PRISM 7900 system (Applied Biosystems) with the following program: $95^{\circ} \mathrm{C}, 10 \mathrm{~min} ; 40$ cycles of $95^{\circ} \mathrm{C}, 10 \mathrm{sec}$ and $60{ }^{\circ} \mathrm{C}, 60 \mathrm{sec}$. The relative expression levels of miRNA and mRNA were normalized to U6 (a ubiquitous small nuclear RNA) and GAPDH, respectively, and calculated using the $2^{-\triangle \Delta \mathrm{Ct}}$ method. Primers for miRNA and mRNA are listed in Table S5.

\section{miRNA target gene prediction}

For miRNAs that were differentially expressed between the $\mathrm{iCD}$ and $\mathrm{HC}$ groups, target genes were scored and screened by combining the algorithms of TargetScan (http://www.targetscan.org), miRBase (http://www.mirbase.org), and miRWalk (http://www.ma.uni-heidelberg.de/apps/zmf/ mirwalk/). 


\section{Luciferase reporter assays}

Mutated 3'UTR gene sequences were inserted into the XbaI and FseI sites of the pGL3 vector

(Genechem, Shanghai, China). HEK293T cells transfected with miRNA mimics (RiboBio, Guangzhou,

China) or a negative control were seeded into 24 -well plates. The pGL3 vector $(0.1 \mu \mathrm{g})$ with the inserted

3'UTR sequence was cotransfected with the pRL-TK vector $(0.02 \mu \mathrm{g})$ using X-treme GENE HP (Roche).

After 24 hours, the cells were harvested according to the manufacturer's protocol (Promega Corporation,

Fitchburg, Wisconsin, USA) and firefly and Renilla luciferase activities were detected using the Dual-Luciferase Reporter Assay System (Promega Corporation, Fitchburg, Wisconsin, USA) with a Tecan Infinite reader. Taget genes and sequence of hsa-miR-16-1 and hsa-miR-142-3p were shown in Table S6.

\section{Data analysis}

miRNAs with significant differences in expression were normalized using the double-filtering criteria: adjusted P-value (FDR) $<0.05$ and absolute $\log 2$ fold change (absolute $\log 2 \mathrm{FC}$ ) $>1$. Pathway and GO analyses, cluster analysis (Cluster 3.0) and TreeView analysis (Stanford University, Stanford, CA, USA) were applied to the results. miRNA and mRNA expression differences were detected and tested using Kruskal-Wallis and Holm-Bonferroni post-hoc tests ${ }^{21}$. Measurement data are expressed as the means \pm standard deviation, and comparisons between two groups were conducted using independent sample t-tests (SPSS17.0, Chicago, IL). Figures were plotted using Graphpad Prism 5 (San Diego, CA, USA). P values of $<0.05$ were indicative of statistically significant differences.

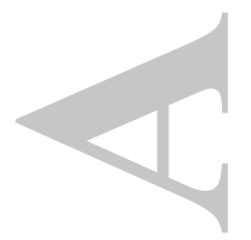




\section{Results}

A comprehensive summary of sample information is listed in Table S1. CD samples were divided into ascending colon mucosal samples with inflammation (iCD) or without inflammation (niCD). The total number of samples included $7 \mathrm{iCD}, 7 \mathrm{niCD}$, and 7 healthy controls. Overall, intestinal mucosa samples (from 14 individuals: seven CD patients and seven healthy volunteers) were obtained (Figure 1A). Specific sample information, including ethnic background, CDAI, previous treatments, and other clinical data, is listed in Table S2. Morphological observation of the patient intestinal mucosal tissues is shown in Figure S1. Figure S1A shows that in the HC group, the epithelium was intact, with no ulcerations. The intestinal glands were arranged in an orderly manner in the lamina propria. Figure S1B shows that in the iCD group, the mucosal epithelium was damaged, with ulcerations and obvious hemorrhage. The intestinal glands were reduced in size. In addition, there was substantial lymphocytic cell infiltration in the intestinal mucosa and submucosa. Figure S1C shows that in the niCD group, there was a small amount of hyperemia and inflammatory cell infiltration in the intestinal mucosa and submucosa. The intestinal glands were arranged in an orderly

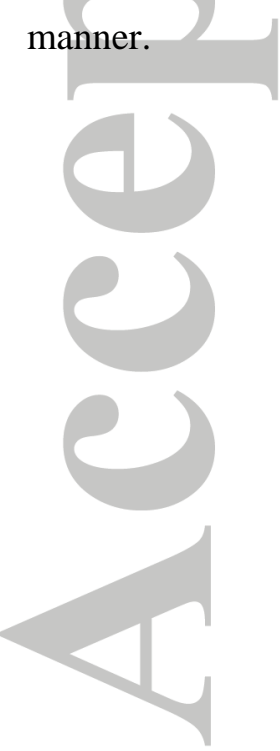


Overlaps in miRNAs differentially expressed between inflammatory and non-inflammatory CD mucosae

The iCD and HC groups had eight differentially expressed miRNAs in common with the niCD and $\mathrm{HC}$ groups and 45 overlaps with the $\mathrm{iCD}$ and niCD groups; the niCD and $\mathrm{HC}$ groups had five differentially expressed miRNAs in common with the $\mathrm{iCD}$ and niCD groups, and the three groups had only one common overlap, hsa-miR-223 (Figure 1B). We compared the expression of the four most significantly upregulated miRNAs (with defined names) (miR-144, miR-451, miR-31, and miR-142-3p) and the four most significantly downregulated miRNAs (miR-1973, miR-1205, miR-5481 and miR-491-3p) in iCD samples compared to HC samples. In inflammatory colonic mucosae of CD patients, miR-144, miR-451, miR-31, and miR-142-3p demonstrated significantly higher expression levels than in healthy colonic mucosae or non-inflamed areas, where the expression levels of these four miRNAs were similar (Figure 1C). In contrast, the expression levels of miR-1973, miR-1205, miR-5481, and miR-491-3p were significantly reduced in the inflammatory colonic mucosae of $\mathrm{CD}$ patients relative to healthy colonic mucosae. The expression of miR-1205 was reduced in both inflammatory and non-inflammatory areas; however, the expression levels of miR-1973, miR-5481, and miR-491-3p in non-inflammatory areas were similar to those of healthy tissues (Figure 1D).

\section{miRNA expression profiles change in inflammatory ascending colon mucosa}

We found that the miRNA expression profile in the ascending colon mucosae differed significantly between the iCD and HC groups. Of the 1257 miRNA detected, 78 miRNAs differed significantly between the iCD and HC groups ( $\mathrm{P}<0.05$, FDR $<0.05)$, with 43 miRNAs upregulated 
and 35 downregulated (Figure 1B, Figure 2B). The heat map of differentially expressed miRNAs suggests that the $\mathrm{CD}$ group of samples differed from the HC group (Figure 2A).

miRNA expression profile changes in non-inflammatory ascending colon mucosae

Our microarray analysis demonstrated that, compared with the HC group, two miRNAs were upregulated and 18 miRNAs were downregulated in the non-inflammatory ascending colon mucosae of CD patients (Figure 1B, Figure 3B). Cluster analyses of the differentially expressed miRNAs suggest differences between samples from the niCD group and those from the $\mathrm{HC}$ group (Figure 3A).

\section{miRNA expression levels differ between inflammatory and non-inflammatory regions of the ascending colon}

We compared the changes in mucosal miRNA expression profiles between the inflammatory and non-inflammatory regions of the ascending colon. Expression and cluster analyses of miRNAs with significant differences revealed differences between the two sample groups. Among the miRNAs differentially expressed in the inflammatory versus non-inflammatory areas, 73 were upregulated and 27 were downregulated (Figure 1B, Figure 4A-B).

\section{mRNA expression profiles of the inflammatory ascending colon in CD patients differ from healthy controls}

Among the 36,333 probes (NimbleGen HG 18.0), 3,370 genes demonstrated significant differences between the $\mathrm{HC}$ and $\mathrm{iCD}$ groups $(\log 2 \mathrm{FC}$ absolute values $>1$ and adjusted $\mathrm{P}$-value < 0.05 ), of which 2,169 were upregulated and 1,201 were downregulated in the iCD group compared to the HC group. The pathways associated with the differentially up- and downregulated genes, and 
genes involved in each pathway are listed in Tables S3 and S4; more signalling pathways were associated with the upregulated genes than the downregulated genes. Pathways of upregulated genes closely related to $\mathrm{CD}$ pathology mainly included antigen processing and presentation, pathogenic Escherichia coli infection, cytokine-cytokine receptor interaction, and intestinal immune network for IgA production. Pathways of downregulated genes closely related to CD pathology mainly included the Hedgehog signalling pathway, pathways in cancer, natural killer cell-mediated cytotoxicity, and the mTOR signalling pathway.

We observed that inflammatory factors and cytokines related to immune functions were dysregulated in the inflammatory ascending colon of CD patients. Among them, ILIRL2, IL17, IL13, and $I L 22 R A 2$ showed varying degrees of downregulation in inflammatory tissues, while $I L-7 R, I L 1 R B$,

IL6R, IL32, CXCL14, IL18BP, and CXCL5 showed varying degrees of upregulation (Figure 5D).

\section{mRNA and miRNA expression is correlated in the HC and iCD groups}

We selected genes with high levels of expression in the inflammatory areas of CD patients and differentially expressed miRNAs to conduct correlation analyses of gene and miRNA expression (Log2 expression). We found that REG4, PLA2G2A, HLA-DQB1 and CXCL5 were highly expressed in the inflammatory areas of $\mathrm{CD}$ patients compared with $\mathrm{HC}(\log 2 \mathrm{FC}$ values $=6.76,5.81,3.87$, and 2.58 , respectively), and these genes highly correlated $\left(r^{2}>0.97\right)$ with different miRNAs. For example, there were positive correlations between REG4 and miR-21 ( $\mathrm{r}^{2}=0.9991$, Figure S3A) and between PLA2G2A and miR-126 ( ${ }^{2}=0.9996$, Figure S3C). Conversely, there were negative correlations between CXCL5 and let-7i $\left(\mathrm{r}^{2}=0.9943\right.$, Figure S3B) and between HLA-DQB1 and miR-106b* $\left(\mathrm{r}^{2}=\right.$ 0.9706, Figure S3D). 


\section{Quantitative real-time PCR validation}

We chose three miRNAs (let-7a, let-7i, and miR-21) that were upregulated in the iCD group (another 7 patients) compared with healthy controls (another 7 individuals) as well as three downregulated miRNAs (miR-491-3p, miR-106b* and let-7d*) to be validated via $q R T-P C R$. The microarray results are shown in Figure 2B. The expression levels of let-7a, let-7i, and miR-21 were significantly increased in inflammatory areas of $\mathrm{CD}$ patients by $4.25,2.5$, and 2.68 -fold, respectively, (Figure 5A); those of miR-491-3p, miR-106b*, and let-7d* were significantly reduced in the inflammatory areas of CD patients by $0.33,0.53$ and 0.44 -fold, respectively (Figure 5C). We also verified the expression of $L I M K 1, T B K B P 1$, and TMEM3OB in healthy control samples and those from inflammatory areas of CD patients. The results showed that, compared with the HC group, the expression levels of $L I M K 1$ and $T B K B P 1$ were reduced in the inflammatory areas of CD by 0.50 and 0.33-fold, respectively, while that of TMEM3OB was upregulated in the inflammatory areas of CD by 4.02-fold; these differences were all significant (Figure 5B).

\section{C10orf54 is a potential target gene of miR-16-1}

Compared with the negative control group, the luciferase assay results of wild-type C10orf54 and SON suggested that miR-16-1 reduced expression by $37 \%(\mathrm{P}=0.004)$ and $20 \%(\mathrm{P}=0.014)$, respectively, suggesting that miR-16-1 could bind to the 3'UTR-1 of these target genes and inhibit their expression. Compared with the corresponding wild-type control groups, the luciferase assay results of the C10orf54 and SON mutant 3'UTR constructs demonstrated that miR-16-1 led to an increase of $23 \%(\mathrm{P}=0.005)$ and a decrease of $3 \%$, respectively. These data indicate that miR-16-1 cannot bind to the mutated C10orf54 3'UTR and thus cannot inhibit its expression (Figure 6B). We 
therefore concluded that miR-16-1 can directly target the 3'UTR-1 region of the C10orf54 target gene to suppress its expression. We further observed a negative correlation between miR-16 expression and the mRNA expression level of C10orf54 $\left(\mathrm{r}^{2}=0.7126\right.$, Figure 6A).

We also investigated the effects of miR-142-3p on the luciferase activities of its predicted target genes ATG16L1 and VPS24. Compared with the negative control group, the luciferase activity results showed reductions in the expression levels of wild-type $A T G 16 L 1$ and VPS24 of $10 \%(\mathrm{P}=0.029)$ and $17 \%(\mathrm{P}=0.031)$, respectively, suggesting that miR-142-3p can bind to the 3'UTR-1 of these target genes and inhibit their expression. Compared with the corresponding wild-type groups, luciferase activity test results of the mutants increased the expression levels of ATG16L1 and VPS24 by $11 \%$ and $10 \%$ in the presence of miR-142-3p; however, these differences were not significant, suggesting that miR-142-3p has weak binding capacities with ATG16L1 and VPS24, through which it can inhibit their expression (Figure S2). 


\section{Discussion}

To our knowledge, the present study is the first to investigate miRNA expression profiles in the ascending colon mucosae of patients with active $\mathrm{CD}$ from the perspective of inflammatory and non-inflammatory tissues. Our results show that inflammatory and non-inflammatory regions of ascending colon mucosae have unique miRNA expression profiles. miRNAs are involved in various biological processes, such as cell differentiation and proliferation, apoptosis, and autophagy, and are closely related to the inflammation-mediated condition of $\mathrm{CD}^{22,23}$.

Previous studies have reported the miRNA expression profiles of inflammatory and non-inflammatory colonic mucosa, of colonic mucosa in active and inactive disease phases, and of peripheral blood ${ }^{13,16}$. Although some immune-related miRNAs have similar expression profiles, most miRNAs have unique expression patterns ${ }^{24,25}$. Previously, using healthy volunteers as controls, we compared the miRNA expression profiles in the mucosae of five sigmoid colon samples affected by $\mathrm{CD}$ and six ileum samples affected with $\mathrm{CD}$. Microarray analysis determined that three miRNAs (miR-106a, miR-23b, and miR-191) were highly expressed and two miRNAs (miR-19b and miR-629) were downregulated in the sigmoid colon samples afflicted with CD, while four miRNAs (miR-16, miR-21, miR-223, and miR-594) were upregulated and no miRNAs were downregulated in the inflamed ileum ${ }^{16}$. In addition, it has been found that miR-495-5p is downregulated in the inflammatory terminal ileum of CD patients, while miR-361-3p was upregulated and let-7b-5p was downregulated in the non-inflammatory terminal ileum ${ }^{15}$. Previous studies in paediatric IBD patients investigated miRNA expression profile changes in the rectum and serum and identified three miRNAs (miR-192, miR-142-3p, miR-21) with significantly upregulated expression ${ }^{17}$. In a study that did not 
distinguish specific areas of the colon, CD patients with colonic inflammation expressed five upregulated miRNAs (miR-9, miR-126, miR-130a, miR-181C, and miR-375) compared to healthy volunteers, while four miRNAs (miR-9*, miR-30a*, miR-30c, and miR-223) were upregulated in non-inflammatory colonic mucosae ${ }^{26}$. The miRNA expression profiles of peripheral blood from two adult CD patients showed significant upregulation of miR-199a-5p, miR-362-3p, and miR-532-3p. In another study, miR-16, miR-23a, miR-29a, miR-106a, miR-107, miR-126, miR-191, and miR-200c were upregulated, while in another study, miR-340* and miRplus-E1271 were upregulated ${ }^{13,27}$. The differentially expressed miRNAs identified from these studies are not exactly the same, which may be due to differing factors such as sample size, CD patient characteristics (age, drug history, with or without surgery, etc.), and subject population demographics (nationality, race) ${ }^{15}$. Although the ascending colon is a site frequently affected by $\mathrm{CD}$, until now, no studies have focused on the miRNA expression profiles of inflammatory and non-inflammatory regions of ascending colon mucosae in CD patients.

1

In our study, we found that hsa-miR-16, hsa-miR-21, and hsa-miR-223 expression levels were increased in the inflammatory ascending colon mucosa, and the results are similar to the expression patterns in inflammatory ileal mucosa ${ }^{16}$, while showing significant differences from inflammatory sigmoid colon mucosa. miR-21 is closely related to myocardial fibrosis, and miR-146b is associated with ischaemia and hypoxia ${ }^{28}$. In addition, miR-144, miR-451, miR-31, and miR-142-3p, which were also upregulated in inflammatory colonic mucosa, demonstrated significant differences in expression between the inflammatory ascending colon mucosa of active CD patients and healthy controls (Log2 FC > 2.6, Figure 1C). Among these, miR-31 has been closely related to inflammation-related colon 
cancer, is dynamically changed ${ }^{29}$, and can act on target genes such as SNAIL, TWIST2, and ZEBI ${ }^{30}$. It has been reported that expression of the miR-29 family is reduced in colonic mucosa associated with small intestinal narrowing; miR-29b inhibits TGF- $\beta$-induced intestinal fibrosis in CD patients ${ }^{31}$, 32 and can directly regulate collagen genes $(C O L 1, C O L 3 \text {, and } C O L 4)^{33} . \quad$ Interestingly, the expression levels of miR-32*, miR-106b*, and let-7d* were reduced in the inflammatory tissues compared to healthy controls and increased compared to the non-inflammatory tissue of the CD ascending colon. Let-7 is involved in the regulation of immune inflammation by regulating the activity of NF- $\mathrm{\kappa B}$ and mediating the downregulation of IL-6 in macrophages ${ }^{34}$.

We analysed the coexpression of miRNAs and mRNAs in the inflammatory ascending colon mucosae of CD patients and in the ascending colon mucosae of healthy volunteers. CXCL5, 14 and 16, and $I L-13,17$, and 32 as well as other chemokines and cytokines closely related to inflammation were all upregulated at sites of colon inflammation (Figure 5D). Among these, CXCL5 and let-7i were negatively correlated (Figure S3B). CXCL14, CXCL5 and IL-17 are all highly expressed in inflammatory tissues and have demonstrated hypermethylation in UC but not CD patients. This feature might be used as a diagnostic marker for these two diseases ${ }^{35}$. During the inflammation phase of IBD, the number of Th17 cells is increased, while $I L-13, I L-17$, and CXCL5 are all upregulated, suggesting that $I L-13, I L-17$, and $C X C L 5$ are closely related to the pathology of enteritis and act closely with the PI3K-AKT signalling pathway ${ }^{36,37}$. IL-32 is a newly discovered proinflammatory cytokine that can activate the $\mathrm{P} 38$ and NF- $\mathrm{KB}$ signalling pathways to play a role in the immune response of IBD, but its specific roles with other proteins are still to be elucidated ${ }^{38}$. It has been reported that $D U O X 2$ and $D U O X A 2$ are upregulated and closely related to inflammation in active UC. 
In our study, compared with healthy volunteers, DUOX2 was upregulated in inflammatory ascending colon mucosae of CD patients ${ }^{39}$, whereas DUOXA2 did not show significant differences. DUOX2 is an NADPH oxidase homologue and can release hydrogen peroxide. Another NADPH oxidase homologue, NOX1, also showed increased expression in iCD. Mesalazine therapy in UC patients acts by inhibiting DUOX2-mediated production of hydrogen peroxide, thereby reducing ROS-induced intestinal mucosal damage ${ }^{39}$. In addition, we observed that, in comparison with healthy volunteers, the pro-inflammatory cytokines demonstrating significant changes in the inflammatory ascending colon, such as REG4, PLA2G2A, and $H L A-D Q B 1$, were also abnormally regulated and significantly upregulated in other inflammatory tissues. REG4 is a gene involved in anti-microbial reactions, and its expression in the inflammatory CD ascending colon was increased 108-fold compared to that of the healthy human; this upregulation was positively correlated with miR-21 (Figure S3A). This result is consistent with previous studies showing that REG4 expression in $\mathrm{CD}$ was only upregulated in comparison with healthy volunteers, and there was no change between $\mathrm{CD}$ and $\mathrm{UC}$ patients ${ }^{40} . R E G 4$ is an important member of the regeneration gene family. It participates in the proliferation and differentiation of intestinal cells and plays an important role in the diagnosis of intestinal tumours.

PLA2G2A is an $\alpha$-defensin specifically expressed in Paneth cells, and its expression level can reflect the number of Paneth cells. Our results show that $P L A 2 G 2 A$ was highly expressed in the ascending colon mucosae of CD patients (56-fold) and was positively correlated with miR-126 (Figure S3C), consistent with previous studies ${ }^{41}$. The human leukocyte antigen (HLA) allelic polymorphism is closely related to the genetic susceptibility of Han people to CD. Our results suggest that $H L A-D Q B 1$ was upregulated in the inflammatory ascending colon, while other members of the $H L A$ gene family 
showed varying degrees of increased expression (Supplementary Material), suggesting that patients have a greater risk of $\mathrm{CD}$ susceptibility; furthermore, the expression of these genes was negatively correlated with miR-106b* (Figure S3D). Based on the prediction of miRNA target genes, we calculated the relevance and selected miRNAs and target genes for luciferase reporter gene assays.

We discovered that miR-16-1 mimics could directly act on the 3'UTR of the wild-type C10orf54, inhibiting its expression. However, the inhibitory effect of the miR-16-1 mimics disappeared following mutation of the C10orf54 3'UTR (Figure 6B), and the two were negatively correlated. Upregulation of miR-16 in the inflammatory areas of the ascending colon mucosa probably inhibits the expression of C10orf54, which in turn causes immune inflammatory responses in the ascending colon, and reduction in miR-16 expression suggests the possibility of canceration of the inflammatory colon $^{42}$. miR-142-3p can act on the autophagy-related CD-susceptibility gene ATG16L1 in the CD ascending colon and play a regulatory role in colonic epithelial autophagy ${ }^{43}$.

We selected a number of miRNAs and validated these miRNAs via qRT-PCR. Compared with healthy volunteers, the expression levels of miR-21, let-7a, and let-7i were significantly upregulated in the inflammatory ascending colon mucosae of adult L2B1 CD patients, while those of miR-491-3p, miR-106b*, and let-7d* were significantly reduced. We also verified the expression of mRNAs and found that $T M E M 30 B$ was significantly upregulated (four-fold) while $L M K 1$ and $T B K B P$ were significantly reduced.

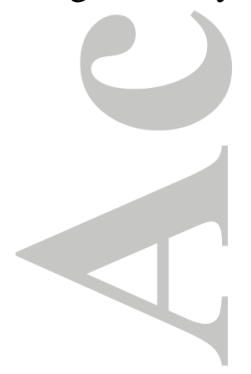


In summary, our data suggest that dysregulation of miRNA expression is mainly restricted to the inflammatory ascending colon mucosa of adult L2B1 CD patients: there are no obvious changes in non-inflammatory ascending colon mucosa, and the characteristics of the different miRNA expression profiles can be used to distinguish whether there is inflammatory infiltration in the colon.

The main limitation is that the differences in the miRNA expression profiles between inflammatory and non-inflammatory ascending colon mucosae may be due to the different cell types collected via biopsy. In addition, the degree of inflammatory infiltration may affect miRNA expression in the inflammatory ascending colon mucosa. It will be important that the relationship between miRNAs and immune inflammation warrants further investigation.

\section{References}

1 Burisch J, Jess T, Martinato M, et al. The burden of inflammatory bowel disease in Europe. $J$ Crohns Colitis 2013; 7:322-337.

2 Wang YF, Ouyang Q, Hu RW. Progression of inflammatory bowel disease in China. J Dig Dis $2010 ; 11: 76-82$

3 Leong RW, Lau JY, Sung JJ. The epidemiology and phenotype of Crohn's disease in the Chinese population. Inflamm Bowel Dis 2004; 10:646-651.

4 Ventham NT, Kennedy NA, Nimmo ER, et al. Beyond gene discovery in inflammatory bowel disease: The emerging role of epigenetics. Gastroenterology 2013; 145:293-308. 
5 Khor B, Gardet A, Xavier RJ. Genetics and pathogenesis of inflammatory bowel disease. Nature $2011 ; 474: 307-317$

6 Jostins L, Ripke S, Weersma RK, et al. Regulation of immune responses and tolerance: The microRNA perspective. Immunol Rev 2013; 253:112-128.

7 Bartel DP. MicroRNAs: genomics, biogenesis, mechanism, and function. Cell 2004; 116:281-297.

8 Xiao C, Rajewsky K. MicroRNA control in the immune system: basic principles. Cell 2009; 136:26-36.

9 Chen CZ, Schaffert S, Fragoso R, et al. Regulation of immune responses and tolerance: the microRNA perspective. Immunol Rev 2013; 253:112-128.

10 Ye D, Guo S, Al-Sadi R, et al. MicroRNA regulation of intestinal epithelial tight junction permeability. Gastroenterology 2011; 141:1323-1333.

11 Brain O, Owens BM, Pichulik T, et al. The intracellular sensor NOD2 induces microRNA-29 expression in human dendritic cells to limit IL-23 release. Immunity 2013; 39:521-536.

12 Brest $\mathrm{P}$, Lapaquette $\mathrm{P}$, Souidi $\mathrm{M}$, et al. A synonymous variant in IRGM alters a binding site for miR-196 and causes deregulation of IRGM-dependent xenophagy in Crohn's disease. Nat Genet 2011; 43:242-245.

13 Wu F, Guo NJ, Tian H, et al. Peripheral blood microRNAs distinguish active ulcerative colitis and Crohn's disease. Inflamm Bowel Dis 2011; 17:241-250.

14 Dalal SR, Kwon JH. The role of microRNA in inflammatory bowel disease. Gastroenterol Hepatol 2010; 6:714-722. 
15 Guo Z, Wu R, Gong J, et al. Altered microRNA expression in inflamed and non-inflamed terminal ileal mucosa of adult patients with active Crohn's disease. J Gastroenterol Hepatol 2015;

\section{0:109-116.}

16 Wu F, Zhang S, Dassopoulos T, et al. Identification of microRNAs associated with ileal and colonic Crohn's disease. Inflamm Bowel Dis 2010; 16:1729-1738.

17 Zahm AM, Hand NJ, Tsoucas DM, et al. Rectal microRNAs are perturbed in paediatric inflammatory bowel disease of the colon. J Crohns Colitis 2014; 8:1108-1117.

18 Gower-Rousseau C, Dauchet L, Vernier-Massouille G, et al. The natural history of pediatric ulcerative colitis: a population-based cohort study. Am J Gastroenterol 2009;104(8):2080-8.

19 Starr AE, Deeke SA, Ning Z, et al. Proteomic analysis of ascending colon biopsies from a paediatric inflammatory bowel disease inception cohort identifies protein biomarkers that differentiate Crohn's disease from UC. Gut 2016; gutjnl-2015-310705. doi: 10.1136/gutjnl-2015-310705.

20 Satsangi J, Silverberg MS, Vermeire S, et al. The Montreal classification of inflammatory bowel disease: Controversies, consensus, and implications. Gut 2006; 55:749-753.

21 Holm S. A simple sequential rejective multiple test procedure. Scand J Stat 1979; 6:65-70.

22 Zhang X, Zuo X, Yang B, et al. MicroRNA directly enhances mitochondrial translation during muscle differentiation. Cell 2014; 158:607-619.

$23 \mathrm{Su} \mathrm{Z}$, Yang Z, Xu Y, et al. MicroRNAs in apoptosis, autophagy and necroptosis. Oncotarget 2015; 6:8474-8490. 
24 Kalla R, Ventham NT, Kennedy NA, et al. MicroRNAs: New players in IBD. Gut 2015; 64:504-517.

25 Pekow JR, Kwon JH. MicroRNAs in inflammatory bowel disease. Inflamm Bowel Dis 2012;

18:187-193.

26 Fasseu M, Tréton X, Guichard C, et al. Identification of restricted subsets of mature microRNA abnormally expressed in inactive colonic mucosa of patients with inflammatory bowel disease. PLoS One 2010; 5:e13160.

27 Paraskevi A, Theodoropoulos G, Papaconstantinou I, et al. Circulating microRNA in inflammatory bowel disease. J Crohns Colitis 2012; 6:900-904.

28 Yang KC, Ku YC, Lovett M, et al. Combined deep microRNA and mRNA sequencing identifies protective transcriptomal signature of enhanced $\mathrm{PI} 3 \mathrm{~K} \alpha$ signalling in cardiac hypertrophy. $J$ Mol Cell Cardiol 2012; 53:101-112.

29 Olaru AV, Selaru FM, Mori Y, et al. Dynamic changes in the expression of microRNA-31 during inflammatory bowel disease-associated neoplastic transformation. Inflamm Bowel Dis 2011; 17:221-231.

30 Díaz-Martín J, Díaz-López A, Moreno-Bueno G, et al. A core microRNA signature associated with inducers of the epithelial-to-mesenchymal transition. J Pathol 2014; 232:319-329.

31 Nijhuis A, Biancheri P, Lewis A, et al. In Crohn's disease fibrosis-reduced expression of the miR-29 family enhances collagen expression in intestinal fibroblasts. Clin Sci (Lond) 2014; 127:341-350. 
32 Romero-Pérez L, López-García MÁ, Díaz-Martín J, et al. ZEB1 overexpression associated with e-cadherin and microRNA-200 downregulation is characteristic of undifferentiated endometrial carcinoma. Mod Pathol 2013; 26:1514-1524.

33 Luna C, Li G, Qiu J, et al. Role of miR-29b on the regulation of the extracellular matrix in human trabecular meshwork cells under chronic oxidative stress. Mol Vis 2009; 15:2488-2497.

34 Iliopoulos D, Hirsch HA, Struhl K. An epigenetic switch involving NF-kappaB, lin28, let-7 microRNA, and IL6 links inflammation to cell transformation. Cell 2009; 139:693-706.

35 Karatzas PS, Mantzaris GJ, Safioleas M, et al. DNA methylation profile of genes involved in inflammation and autoimmunity in inflammatory bowel disease. Medicine (Baltimore) 2014; 93:e309.

36 Brown JB, Cheresh P, Goretsky T, et al. Epithelial phosphatidylinositol-3-kinase signalling is required for $\beta$-catenin activation and host defense against Citrobacter rodentium infection. Infect Immun 2011; 79:1863-1872.

37 Ślebioda TJ, Bojarska-Junak A, Stanisławowski M, et al. TL1a as a potential local inducer of IL17a expression in colon mucosa of inflammatory bowel disease patients. Scand J Immunol 2015; 82: 352-360.

38 Felaco P, Castellani ML, De Lutiis MA, et al. Il-32: A newly-discovered proinflammatory cytokine. J Biol Regul Homeost Agents 2009; 23:141-147.

39 MacFie TS, Poulsom R, Parker A, et al. DUOX2 and DUOXA2 form the predominant enzyme system capable of producing the reactive oxygen species $\mathrm{H} 2 \mathrm{O} 2$ in active ulcerative colitis and are modulated by 5-aminosalicylic acid. Inflamm Bowel Dis 2014; 20:514-524. 
40 Bjerrum JT, Nyberg C, Olsen J, et al. Assessment of the validity of a multigene analysis in the diagnostics of inflammatory bowel disease. J Intern Med 2014; 275:484-493.

41 Simms LA, Doecke JD, Walsh MD, et al. Reduced alpha-defensin expression is associated with inflammation and not NOD2 mutation status in ileal Crohn's disease. Gut 2008; 57:903-910.

42 Qian J, Jiang B, Li M, et al. Prognostic significance of microRNA-16 expression in human colorectal cancer. World J Surg 2013; 37:2944-2949.

43 Zhai Z, Wu F, Dong F, et al. Human autophagy gene ATG1611 is post-transcriptionally regulated by miR142-3p. Autophagy 2014; 10:468-479.
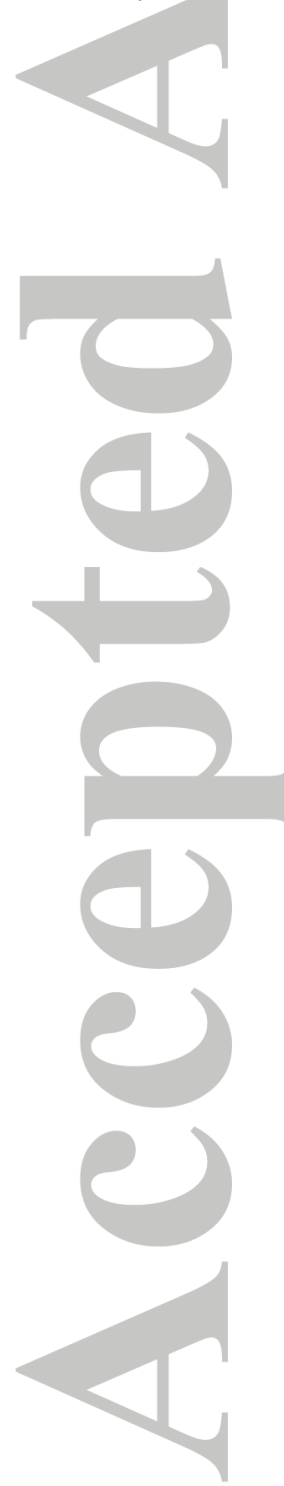
A

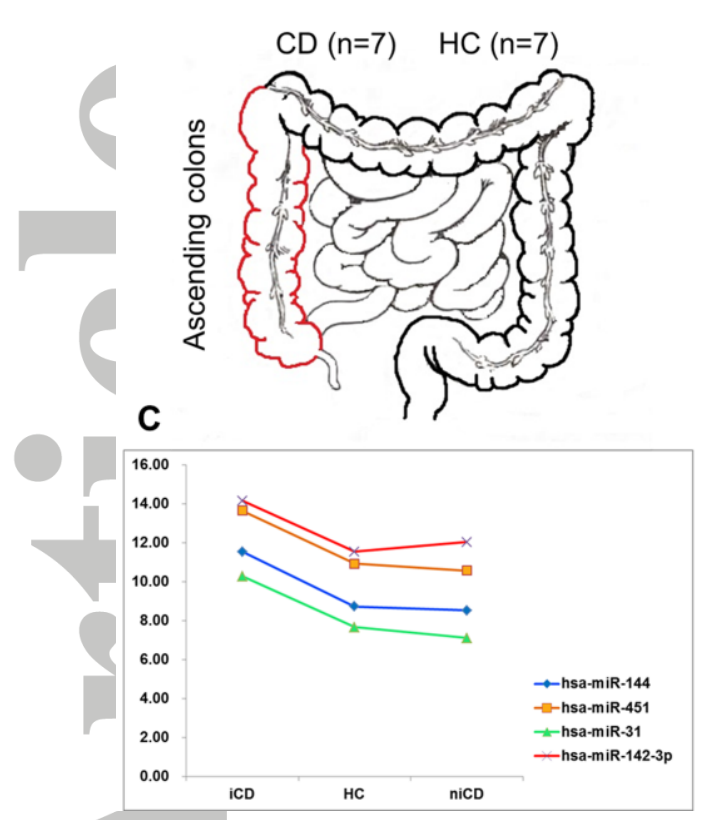

B Differentially expressed miRNAs

(C) 2007 - 2015 Tim Hulsen iCD vs $\mathrm{HC}$

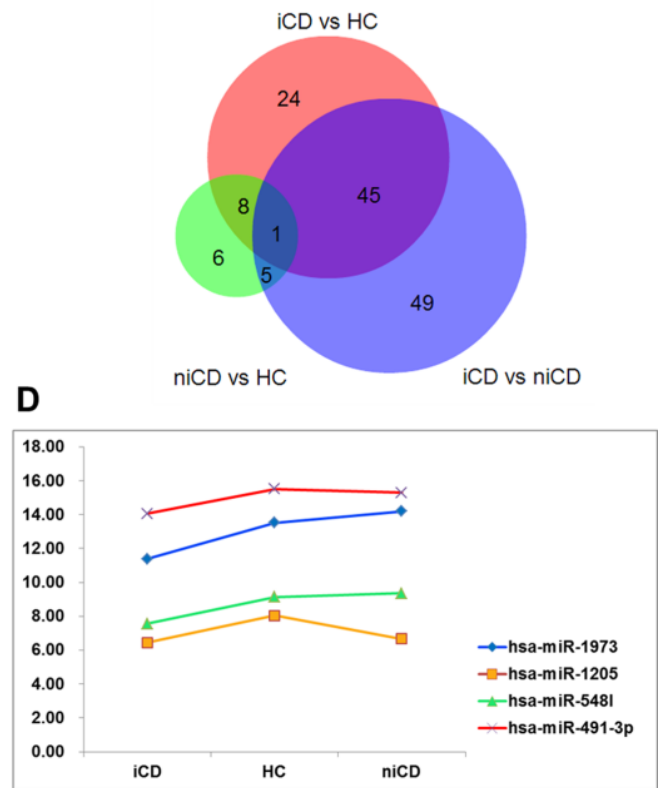

Figure 1 Expression characteristics of miRNAs with differential expression in the ascending colon of $C D$ patients. (A) Biopsy samples from $C D$ patients were all from the ascending colon. (B) Venn diagram with red representing the number of miRNAs differing between $\mathrm{ICD}$ and $\mathrm{HC}$; green representing the number of miRNAs differing between niCD and $\mathrm{HC}$; and blue representing the number of miRNAs differing between iCD and niCD. (C) Respective expression of four miRNAs significantly upregulated in iCD compared with HC. (D) Respective expression of four miRNAs significantly downregulated in iCD compared with HC. 


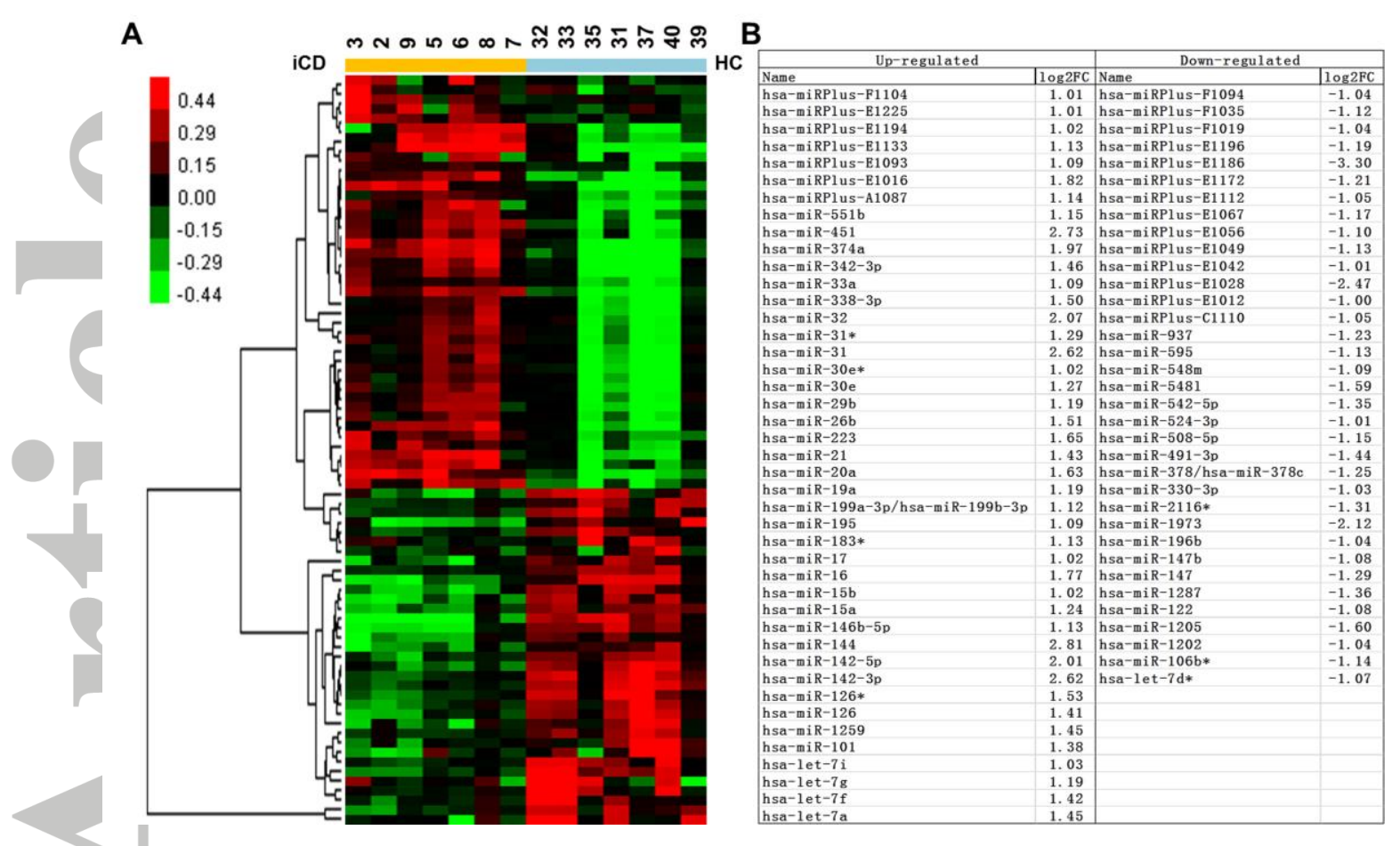

Figure 2 Different miRNA expression profiles between iCD and HC. (A) Heat map with intuitive reflection of the features of expression profile changes in the $\mathrm{ICD}$ and $\mathrm{HC}$ groups. (B) miRNAs

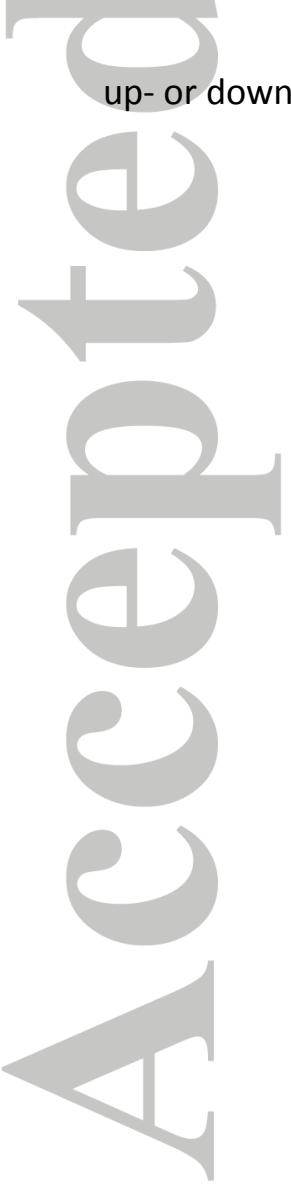

This article is protected by copyright. All rights reserved. 
A

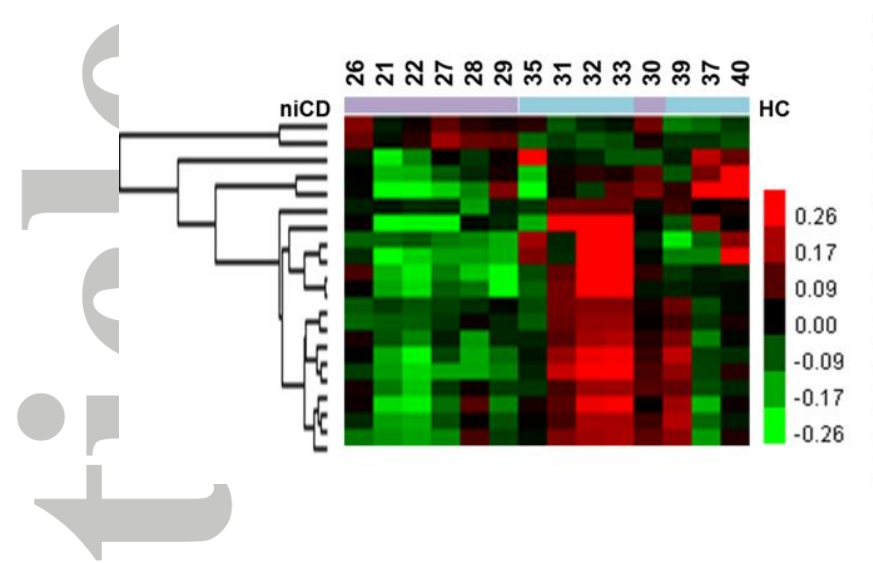

B

\begin{tabular}{|l|l|l|l|}
\hline \multicolumn{2}{|c|}{ Up-regulated } & \multicolumn{2}{c|}{ Down-regulated } \\
\hline Name & log2FC & Name & log2FC \\
\hline hsa-miRPlus-F1195 & 1.02 & hsa-miRPlus-E1186 & -3.99 \\
hsa-miR-150 & 1.17 & hsa-miRPlus-E1049 & -1.07 \\
\hline & & hsa-miR-508-5p & -1.42 \\
\hline & hsa-miR-3176 & -1.06 \\
\hline & hsa-miR-223 & -1.38 \\
\hline & hsa-miR-215 & -1.01 \\
\hline & hsa-miR-2116* & -1.34 \\
\hline & hsa-miR-125b & -1.10 \\
\hline & hsa-miR-1205 & -1.48 \\
\hline & hsa-miR-10a & -1.03 \\
\hline & hsa-miR-563 & -1.00 \\
\hline & hsa-miR-429 & -1.01 \\
\hline & hsa-miR-378/hsa-miR-378c & -1.16 \\
\hline & hsa-miR-3176 & -1.06 \\
\hline & hsa-miR-23a & -1.09 \\
\hline & hsa-miR-222 & -1.00 \\
& hsa-miR-196b & -1.11 \\
& hsa-miR-143* & -1.00 \\
\hline
\end{tabular}

Figure 3 Different miRNA expression profiles between niCD and HC samples. (A) Heat map with

intuitive reflection of the features of expression profile changes in the niCD and $\mathrm{HC}$ groups. (B)

miRNAs up- or downregulated in niCD compared to $H C$ (absolute value $\log 2 F C \geq 1, p<0.05$ ).

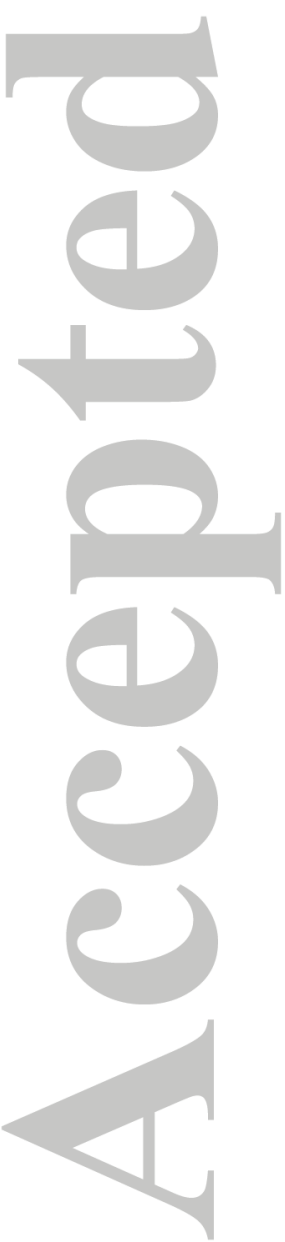

This article is protected by copyright. All rights reserved. 


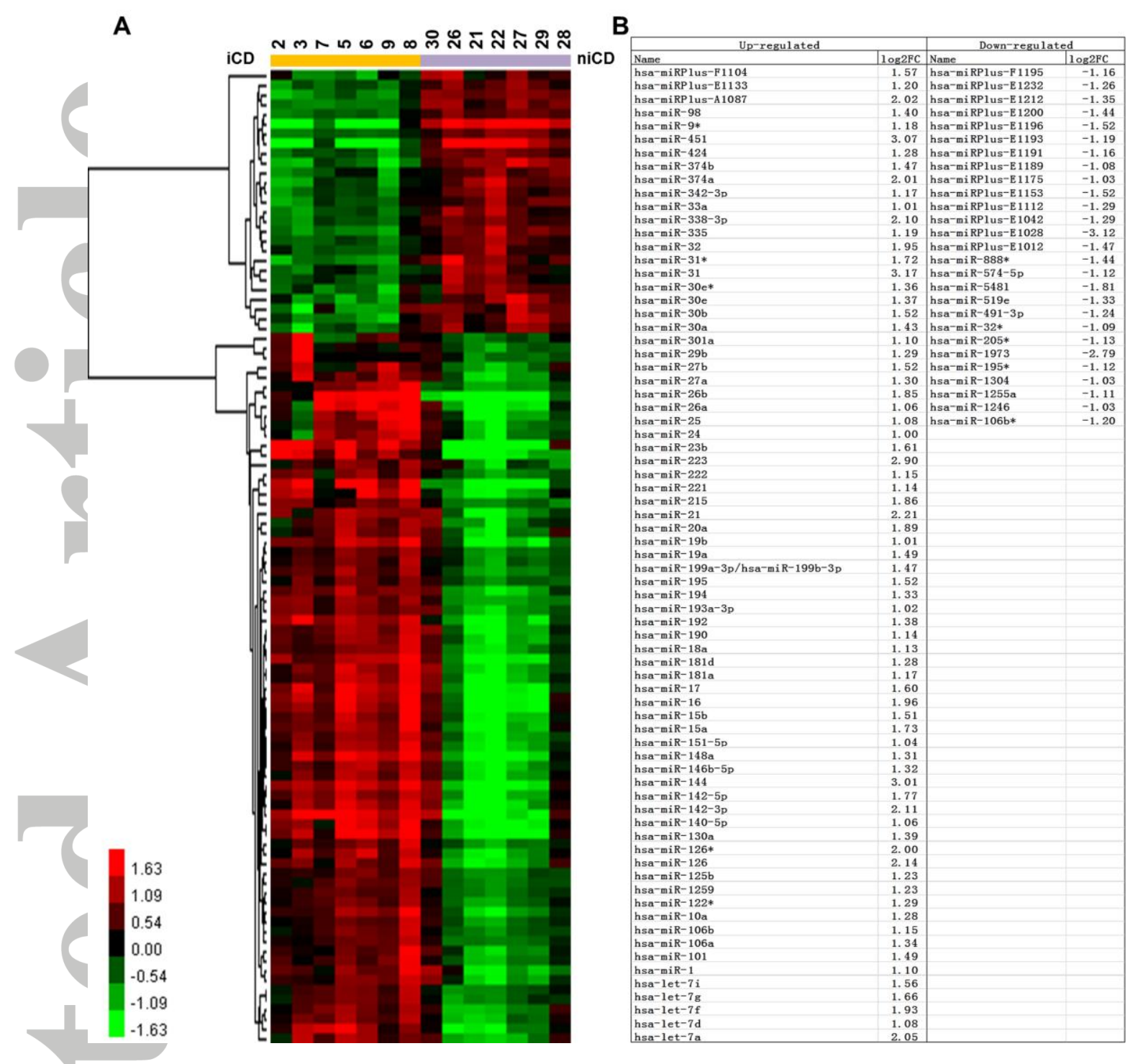

Figure 4 The expression profiles of miRNAs differing between $i C D$ and niCD. (A) Heat map with intuitive reflection of the features of expression profile changes in the $\mathrm{ICD}$ and niCD groups. (B) Up- and downregulated miRNAs comparing iCD to niCD (absolute value $\log 2 F C \geq 1, p<0.05$ ).

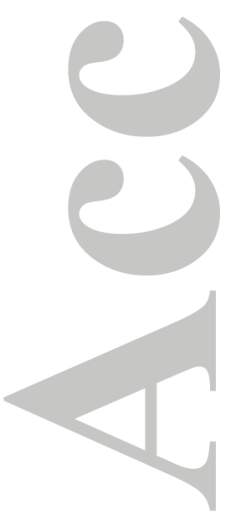

This article is protected by copyright. All rights reserved. 
A

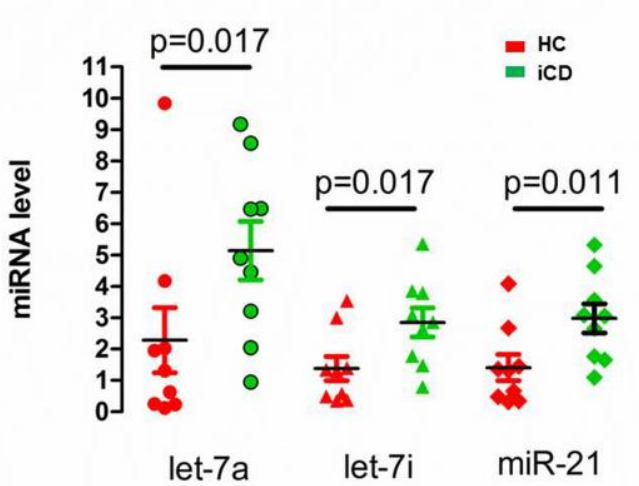

C

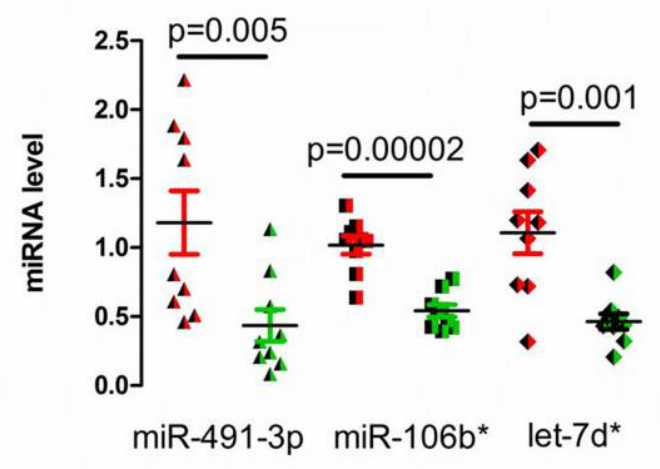

B

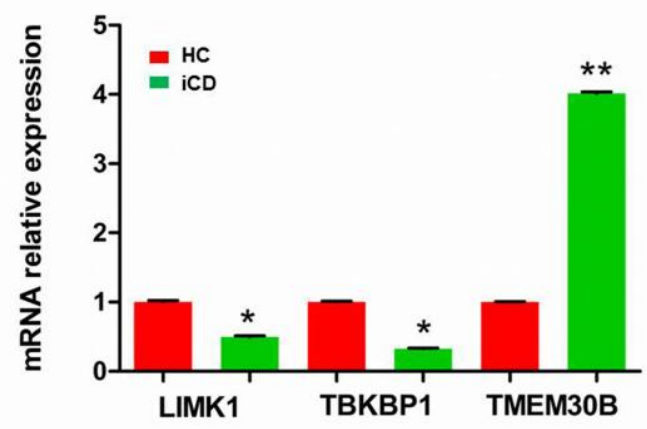

D

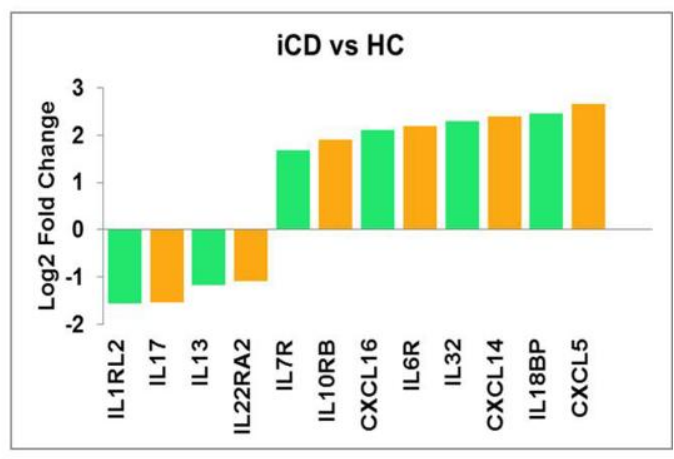

Figure 5 qRT-PCR validation of miRNAs and mRNAs in $\mathrm{iCD}$ and $\mathrm{HC}$ ascending colon mucosae (A) miRNAs upregulated in iCD compared to HC; (B) genes differentially expressed between iCD and $\mathrm{HC}$; (C) miRNAs downregulated in $\mathrm{iCD}$ compared to $\mathrm{HC}$; (D) $\mathrm{iCD} / \mathrm{HC} \log 2 \mathrm{FC}$ ratios of inflammatory cytokines.

This article is protected by copyright. All rights reserved. 

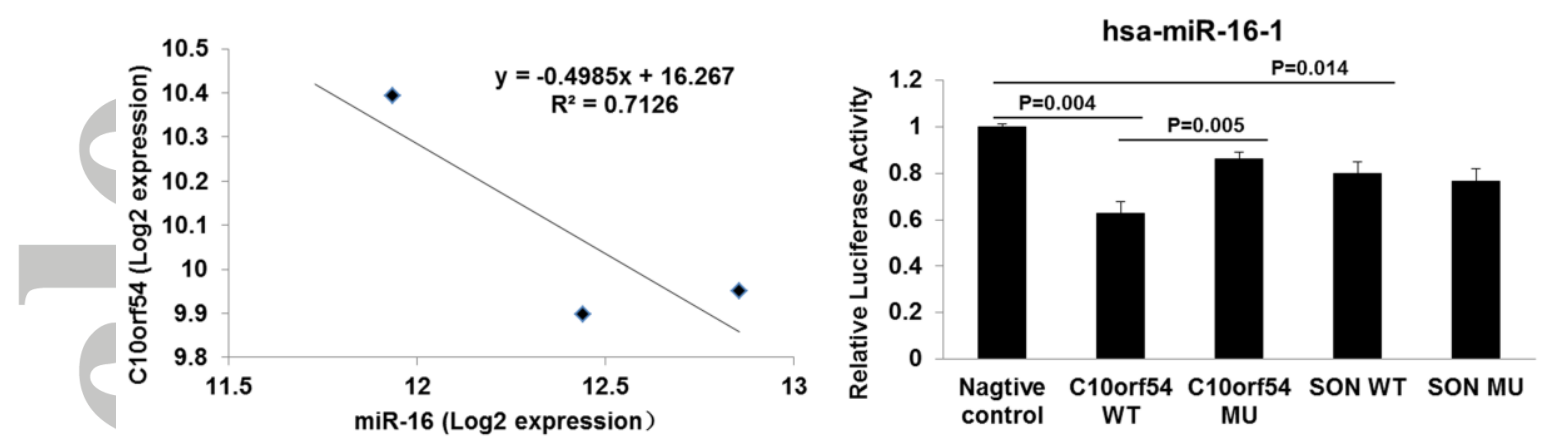

Figure 6 Correlation between C10orf54 and miR-16 expression and luciferase reporter gene assay results. (A) C10orf54 and miR-16 are negatively correlated; (B) hsa-miR-16-1 inhibits wild-type (WT) C10orf54 expression and not mutant C10orf54 (MU) expression.
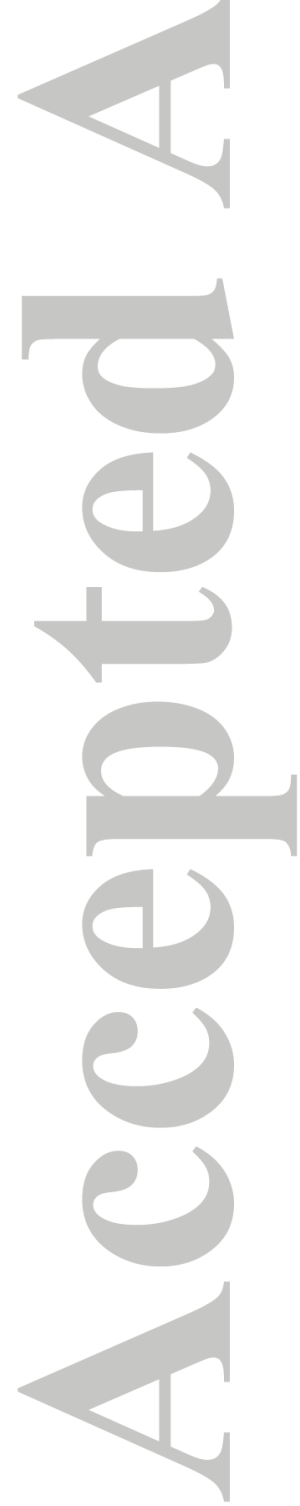

This article is protected by copyright. All rights reserved. 